\title{
Dysphagia in Tongue Cancer Patients
}

\author{
Yu Ri Son, MD, Kyoung Hyo Choi, MD, PhD, Tae Gyun Kim, MD \\ Department of Rehabilitation Medicine, Asan Medical Center, University of Ulsan College of Medicine, Seoul, Korea
}

Objective To identify risk factors for dysphagia in tongue cancer patients. Dysphagia is a common complication of surgery, radiotherapy, and chemotherapy in tongue cancer patients. Previous studies have attempted to identify risk factors for dysphagia in patients with head and neck cancer, but no studies have focused specifically on tongue cancer patients.

Methods This study was conducted on 133 patients who were diagnosed with tongue cancer and who underwent a videofluoroscopy swallowing study (VFSS) between January 2007 and June 2012 at the Asan Medical Center. Data collected from the VFSS were analyzed retrospectively. Patients with aspiration were identified.

Results Patients showed a higher incidence of inadequate tongue control, inadequate chewing, delayed oral transit time, aspiration or penetration, vallecular pouch and pyriform residue, and inadequate laryngeal elevation after surgery. Moreover, male gender, extensive tumor resection, a higher node stage, and more extensive lymph node dissection were major risk factors for aspiration in tongue cancer patients.

Conclusion Tongue cancer patients have difficulties in the pharyngeal phase as well as the oral phase of swallowing. These difficulties can worsen after tongue cancer surgery. Gender, the extent of tumor resection, and lymph node metastasis affect swallowing in tongue cancer patients. Physicians should take these risk factors into account when administering swallowing therapy to tongue cancer patients.

Keywords Head and neck cancer, Tongue cancer, Dysphagia, Aspiration, Videofluoroscopic swallowing study

\section{INTRODUCTION}

Head and neck cancer (HNC) can arise in anatomical regions related to swallowing, but the incidence of aspiration varies [1]. Aspiration can occur not only due to the tumor itself, but also due to surgery, radiotherapy, and

Received June 17, 2014; Accepted September 29, 2014

Corresponding author: Kyoung Hyo Choi

Department of Rehabilitation Medicine, Asan Medical Center University of Ulsan College of Medicine, 88 Olympic-ro 43-gil, Songpa-gu, Seoul 138-736, Korea

Tel: +82-2-3010-3800, Fax: +82-2-3010-6964, E-mail: khchoi@amc.seoul.kr

(c) This is an open-access article distributed under the terms of the Creative Commons Attribution Non-Commercial License (http://creativecommons. org/licenses/by-nc/3.0) which permits unrestricted noncommercial use, distribution, and reproduction in any medium, provided the original work is properly cited.

Copyright $\odot 2015$ by Korean Academy of Rehabilitation Medicine chemotherapy [2]. Because dysphagia affects morbidity and mortality in HNC, it is important that its risk factors be identified so that it can be carefully monitored in clinical practice. Some studies have attempted to identify the risk for aspiration in patients with HNC $[3,4]$. The few studies that have been conducted on dysphagia in HNC patients have focused on HNC subtypes other than tongue cancer. HNC contains several cancer subtypes with different anatomical lesions. Because their characteristics are heterogeneous, it is not easy to predict risk factors for dysphagia in the entire population of HNC patients.

Tongue cancer is the most frequent intraoral HNC. In oral cancer, dysphagia is caused by extensive tissue loss, limited excursion of the remaining tissue, and sensory 
paralysis of the tongue, soft palate, and pharynx [5]. The swallowing function in HNC patients may be affected by the degree of resection and the nature of reconstruction. Although the oral stage of the swallow is generally more severely impaired, the pharyngeal stage of the swallow may be affected if resection includes the tongue base $[6,7]$. Previous studies on dysphagia in oral cancer patients were conducted on a small sample size or lacked radiologic evaluation. A videofluoroscopic swallowing study (VFSS) to evaluate aspiration is needed in patients with HNC who have these risk factors [3].

A VFSS of the swallowing process was conducted to identify risk factors for dysphagia in patients with tongue cancer. Dysphagia characteristics were also compared before and after surgery.

\section{MATERIALS AND METHODS}

\section{Comparison of VFSS findings before and after surgery}

This study was conducted on 133 patients who were diagnosed with tongue cancer and who underwent VFSS between January 2007 and June 2012 at the Asan Medical Center (AMC), Seoul, Korea. VFSS was administered to 87 patients after surgery and to 74 patients prior to surgery. The mean day of VFSS after surgery was $130.1 \pm 25.5$ days.

Patients with neurological disease or head and neck trauma that could influence swallowing function were excluded. Pregnant females were also excluded due to radiation exposure during VFSS. The baseline characteristics of the patient population are shown in Table 1 . The mean age of the patients was 53.5 years. There were 85 men (63.9\%) and 48 women (36.1\%). Tumors were located on the right side in 67 patients $(50.4 \%)$. Surgery was performed on 126 patients (94.7\%). Hemiglossectomy was performed on 16 patients $(12.0 \%)$ and wide resection was performed on 82 patients (61.8\%). Partial glossectomy was performed on 23 patients $(17.3 \%)$ and total glossectomy was performed on five patients (3.8\%). Sixtyone patients (45.9\%) underwent supraomohyoid neck dissection (SOND) and 59 patients (44.5\%) underwent modified radical neck dissection (MRND). In total, 81 patients (61.9\%) underwent reconstruction surgery, which consisted of radial forearm free flap (RFFF) (46 patients, $34.6 \%$ ), medial sural artery perforator free flap (MSP FF) (8 patients, 6.0\%), anterolateral thigh free flap (ATL FF) (24 patients, $18.0 \%$ ), or pectoralis major musculocutane-
Table 1. Baseline characteristics

\begin{tabular}{|c|c|c|c|}
\hline Characteristic & $\begin{array}{c}\text { Total } \\
(n=133)\end{array}$ & $\begin{array}{l}\text { Before } \\
(n=74)\end{array}$ & $\begin{array}{c}\text { After } \\
(\mathbf{n}=\mathbf{8 7})\end{array}$ \\
\hline Gender (male:female) & $85: 48$ & $47: 27$ & $54: 33$ \\
\hline Age (yr) & $53.5 \pm 15$ & $56.0 \pm 15.9$ & $51.5 \pm 14.3$ \\
\hline Side (right:left) & $67: 66$ & $41: 33$ & $41: 46$ \\
\hline \multicolumn{4}{|l|}{ Resection } \\
\hline No resection & $7(5.3)$ & $3(4.1)$ & $4(4.6)$ \\
\hline Partial glossectomy & $105(79.0)$ & $56(75.7)$ & $69(79.3)$ \\
\hline Hemiglossectomy & $16(12.0)$ & $12(16.2)$ & $10(11.5)$ \\
\hline Total glossectomy & $5(3.8)$ & $3(4.1)$ & $4(4.6)$ \\
\hline \multicolumn{4}{|l|}{ Lymph node dissection } \\
\hline None & $13(9.8)$ & $7(9.5)$ & $8(9.2)$ \\
\hline SOND & $61(45.9)$ & $31(41.9)$ & $37(42.5)$ \\
\hline MRND & $59(44.4)$ & $36(44.6)$ & $42(48.3)$ \\
\hline \multicolumn{4}{|l|}{ Reconstruction } \\
\hline None & $52(39.1)$ & $31(41.9)$ & $29(33.3)$ \\
\hline RFFF & $46(34.6)$ & $28(37.8)$ & $27(31.0)$ \\
\hline MSP FF & $8(6.0)$ & $2(2.7)$ & $8(9.2)$ \\
\hline ALT FF & $24(18.0)$ & $12(16.2)$ & $20(23.0)$ \\
\hline PMMC flap & $3(2.3)$ & $1(1.4)$ & $3(3.4)$ \\
\hline \multicolumn{4}{|l|}{ Tumor stage } \\
\hline $\mathrm{T} 1$ & $38(28.6)$ & $18(24.3)$ & $22(25.3)$ \\
\hline $\mathrm{T} 2$ & $40(30.1)$ & $21(28.4)$ & $27(31.0)$ \\
\hline T3 & $3(2.3)$ & $3(4.1)$ & $3(3.4)$ \\
\hline $\mathrm{T} 4$ & $52(39.1)$ & $32(43.2)$ & $35(40.2)$ \\
\hline \multicolumn{4}{|l|}{ Nodal stage } \\
\hline No & $79(59.4)$ & $41(55.4)$ & $53(60.9)$ \\
\hline $\mathrm{N} 1$ & $15(11.3)$ & $7(9.5)$ & $10(11.5)$ \\
\hline N2 & $39(28.6)$ & $25(33.8)$ & $24(27.6)$ \\
\hline N3 & $1(0.8)$ & $1(1.4)$ & $0(0)$ \\
\hline Radiotherapy & $70(52.6)$ & $42(56.8)$ & $47(54.0)$ \\
\hline Chemotherapy & $57(42.9)$ & 35 (47.3) & $37(42.5)$ \\
\hline
\end{tabular}

Values are presented as mean \pm standard deviation or number (\%).

SOND, supraomohyoid neck dissection; MRND, modified radical neck dissection; RFFF, radial forearm free flap; MSP FF, medial sural artery perforator free flap; ATL $\mathrm{FF}$, anterolateral thigh free flap; PMMC flap, pectoralis major musculocutaneous flap.

ous (PMMC) flap (3 patients, 2.3\%). Regarding the tumor node metastasis (TNM) stage, 38 patients $(28.6 \%), 40$ (30.1\%), 3 (2.3\%), and 52 (39.1\%) were classified as $\mathrm{T} 1, \mathrm{~T} 2$, $\mathrm{T} 3$, and T4, respectively. Seventy-nine patients (59.4\%), $15(11.3 \%), 39(28.6 \%)$, and $1(0.8 \%)$ were classified as 
N0, N1, N2, and N3, respectively. Radiotherapy was administered to 70 patients (52.6\%) and chemotherapy was administered to 57 patients $(42.9 \%)$.

To evaluate swallowing function, VFSS was performed using the AMC dysphagia diet (AMCDD), in which $80 \mathrm{~g}$ of AMCDD I (soup), II (chopped fruits) or III (steamed eggs) was mixed with $10 \mathrm{~g}$ of barium sulfate (Solotop Suspension 140; Teajoon Pharm, Seoul, Korea). The thin fluid was a $35 \% \mathrm{v} / \mathrm{v}$ low-concentration liquid diet containing $300 \mathrm{~mL}$ of normal saline mixed with $140 \mathrm{~g} / 100 \mathrm{~mL}$ of barium sulfate. The thick fluid was a $70 \% \mathrm{v} / \mathrm{v}$ high-concentration liquid diet. VFSS was performed on a lateral view using digitalized fluoroscopy (IRF-850-150; Philips Healthcare, Best, The Netherlands) and images were recorded in real time after administration of $5 \mathrm{~mL}$ of each fluid material [8]. Abnormalities in the swallowing process were evaluated in detail using the recorded images.

Patients were observed during the oral, pharyngeal, and esophageal phases of swallowing. Aspiration was defined as the bolus passing through the vocal cords once. Penetration was considered to occur when a bolus entered the glottis and moved as far as the vestibule above the true vocal folds [9].

Risk factors associated with aspiration in tongue cancer patients after surgery

VFSS was administered to 87 patients after surgery among total 133 patients. Baseline characteristics, including age, gender, lesion location (side of the tongue), tumor excision range, extent of reconstruction, TNM staging, and the type of surgery were analyzed as potential risk factors for aspiration after surgery.

For the statistical analysis of age as a risk factor, patients $<65$ years were grouped together and analyzed in comparison to those aged $\geq 65$ years [10]. To evaluate the extent of tumor resection, patients were classified as having received a partial, hemi-, or total glossectomy. Patients who underwent SOND or MRND were evaluated in comparison to those who did not undergo lymph node dissection. Finally, T1 and T2 tumors were distinguished from T3 and T4 tumors, and N0 stage lymph nodes were distinguished from $\mathrm{N} 1$ and $\mathrm{N} 2$ nodes.

\section{Statistical analysis}

All statistical data were analyzed with SPSS ver. 18.0 for Windows (SPSS Inc., Chicago, IL, USA). A chi-square test and Fisher exact test were used to compare the VFSS results before and after surgery. Univariate and multivariate analyses were used to evaluate risk factors for aspiration. Results were considered statistically significant if the $\mathrm{p}$-value was $<0.05$.

\section{RESULTS}

\section{VFSS findings}

VFSS was performed on 74 patients before surgery and on 87 patients after surgery (Table 2). In the oral phase of swallowing, there were no patients with inadequate lip movement before surgery. Patients who underwent VFSS after surgery were observed to have lip movement abnormalities, but this was not statistically significant. In the oral phase, the differences in tongue control, chewing, and oral transit time in patients who were analyzed by VFSS before and after surgery were statistically significant.

Table 2. Comparison of videofluoroscopic swallowing study findings before and after surgery in tongue cancer patients

\begin{tabular}{|c|c|c|c|}
\hline Finding & $\begin{array}{l}\text { Before } \\
(n=74)\end{array}$ & $\begin{array}{c}\text { After } \\
(\mathbf{n}=\mathbf{8 7})\end{array}$ & p-value \\
\hline \multicolumn{4}{|l|}{ Oral phase } \\
\hline Inadequate lip movement & $0(0)$ & $3(3.4)$ & 0.15 \\
\hline Inadequate tongue control & $18(24.3)$ & $64(73.6)$ & $0.00^{*}$ \\
\hline Inadequate chewing & $5(6.8)$ & $25(28.7)$ & $0.00^{*}$ \\
\hline Delayed oral transit time & $3(4.1)$ & $28(32.2)$ & $0.00^{*}$ \\
\hline \multicolumn{4}{|l|}{ Pharyngeal phase } \\
\hline Aspiration or penetration & $8(10.8)$ & $26(29.9)$ & $0.00^{*}$ \\
\hline Fluid aspiration & $15(20.3)$ & $36(41.4)$ & $0.00^{*}$ \\
\hline Solid aspiration & $5(6.8)$ & $15(17.2)$ & 0.05 \\
\hline Nasal regurgitation & $0(0)$ & $4(4.6)$ & 0.07 \\
\hline Vallecular pouch residue & $6(8.1)$ & $39(44.8)$ & $0.00^{*}$ \\
\hline Pyriform sinus residue & $3(4.0)$ & $16(18.4)$ & $0.00^{*}$ \\
\hline $\begin{array}{l}\text { Inadequate laryngeal } \\
\text { elevation }\end{array}$ & $1(1.4)$ & $12(13.8)$ & $0.00^{*}$ \\
\hline Esophageal phase & $0(0)$ & $0(0)$ & - \\
\hline ASHA NOMS rating & $4.95 \pm 2.95$ & $2.82 \pm 2.78$ & $0.00^{*}$ \\
\hline CDS rating & $31.07 \pm 4.94$ & $25.14 \pm 17.09$ & $0.00^{*}$ \\
\hline
\end{tabular}

ASHA NOMS, American Speech-Language-Hearing Association National Outcome Measurement System; CDS, clinical dysphagia scale.

${ }^{*} \mathrm{p}<0.05$ by chi-square test, Fisher exact test. 
In the pharyngeal phase, aspiration or penetration was observed in eight patients (10.8\%) who underwent VFSS before surgery, and in 26 patients $(29.9 \%)$ who underwent VFSS after surgery. Among patients with aspiration, fluid aspiration was observed significantly higher in patients before surgery than after surgery. Solid aspiration showed significant differences between the two groups. While four patients $(4.6 \%)$ showed nasal regurgitation after surgery, nasal regurgitation was not observed in any patient before surgery. Vallecular pouch residue was observed in six patients (8.1\%) before surgery and in 39 patients $(44.8 \%)$ afterward. Pyriform sinus residue was observed in three patients (4.0\%) before surgery and in 16 (18.4\%) after surgery. Inadequate laryngeal elevation was observed in only one (1.4\%) patient who received VFSS before surgery, but was seen in 12 (13.8\%) patients who underwent VFSS after surgery. Among the pharyngeal phase abnormalities, aspiration and penetration, fluid aspiration, vallecular pouch residue, pyriform sinus residue, inadequate laryngeal elevation, and epiglottic closure were statistically different between the two groups.

No patients had esophageal phase abnormalities, either before or after surgery. The rating on the American Speech-Language-Hearing Association (ASHA) National Outcome Measurement System (NOMS) swallowing level scale was $4.95 \pm 2.95$ in the patients who underwent VFSS before surgery and $2.82 \pm 2.78$ in those who underwent VFSS afterward. The clinical dysphagia scale rating was $31.07 \pm 4.94$ before surgery and $25.14 \pm 17.09$ after surgery. Both the ASHA NOMS and clinical dysphagia scale (CDS) ratings were significantly different between the two groups. These findings were not different according to the period of time between the surgeries and when the VFSS was conducted.

VFSS was performed on 28 patients both before and after surgery. Table 3 shows the proportion of patients exhibiting each impairment after surgery, though none of the patients exhibited any of these impairments prior to surgery. Abnormal tongue control was observed in 10 patients $(35.7 \%)$. Chewing abnormalities developed in five patients (17.9\%), and delayed oral transit time was observed in nine patients $(32.1 \%)$. In the pharyngeal phase, eight patients (28.6\%) showed newly developed aspiration or penetration. Vallecular pouch residue was observed in 11 patients (39.3\%), and pyriform sinus residue and aggravation of laryngeal elevation emerged in
Table 3. Aggravation of videofluoroscopic swallowing study findings before and after surgery

\begin{tabular}{lcc}
\hline & \multicolumn{2}{c}{ Impairment } \\
\cline { 2 - 3 } & No. (\%) & CI \\
\hline Oral phase & $1(3.6)$ & - \\
Inadequate lip movement & $10(35.7)$ & $0.354-0.822$ \\
Inadequate tongue control & $5(17.9)$ & $0.045-0.371$ \\
Inadequate chewing & $9(32.1)$ & $0.172-0.548$ \\
Delayed oral transit time & & \\
Pharyngeal phase & $8(28.6)$ & $0.215-0.674$ \\
Aspiration or penetration & $1(3.6)$ & - \\
Nasal regurgitation & $11(39.3)$ & $0.274-0.682$ \\
Vallecular pouch residue & $5(17.9)$ & $0.043-0.357$ \\
Pyriform sinus residue & $5(17.9)$ & $0.039-0.332$ \\
Inadequate laryngeal elevation & $5(0)$ & - \\
\hline Esophageal phase & $0(0)$ &
\end{tabular}

CI, confidence interval.

five patients (17.9\%).

Risk factors associated with aspiration in tongue cancer patients after surgery

Table 4 shows univariate and multivariate analyses of risk factors for aspiration after surgery. Aspiration occurred at a significantly higher rate in males, patients with left tongue tumors, patients who underwent more extensive tumor resection surgery (hemi- or total glossectomy), patients with lymph node metastasis (N1 or N2), and patients who required more extensive lymph node dissection (MRND). However, age, extent of reconstruction, tumor stage, radiotherapy, and chemotherapy had no significant impact on the aspiration risk in patients after surgery.

Multivariate logistic regression analyses revealed that male gender, hemi- or total glossectomy, lymph node metastasis (N1 or N2), and more extensive lymph node dissection (MRND) put patients after surgery at a higher risk of developing aspiration. The incidence of aspiration was 4.43 times higher in male patients than in female patients (hazard ratio [HR], 4.435; 95\% confidence interval [CI], 1.53-12.78). The incidence of aspiration was 6.10 times higher in patients who required hemi- or total glossectomy than in those who only required partial glossectomy (HR, 6.103; 95\% CI, 1.34-27.64). The incidence of aspiration was 1.65 times higher in patients who un- 
Table 4. Univariate and multivariate analyses for risk factors related to aspiration after surgery

\begin{tabular}{|c|c|c|c|c|}
\hline \multirow{2}{*}{ Variable } & \multicolumn{2}{|c|}{ Univariate } & \multicolumn{2}{|c|}{ Multivariate } \\
\hline & $\%$ & p-value & HR (95\% CI) & p-value \\
\hline \multicolumn{5}{|l|}{ Gender } \\
\hline Female & 37.9 & & & \\
\hline Male & 62.1 & $0.00 *$ & $4.43(01.53-12.78)$ & $0.01^{*}$ \\
\hline \multicolumn{5}{|l|}{ Age (yr) } \\
\hline$<65$ & 75.9 & & & \\
\hline$\geq 65$ & 24.1 & 0.57 & & \\
\hline \multicolumn{5}{|l|}{ Side } \\
\hline Right & 47.1 & & & \\
\hline Left & 52.9 & $0.03^{*}$ & $0.35(0.12-1.06)$ & 0.06 \\
\hline \multicolumn{5}{|l|}{ Resection } \\
\hline Partial glossectomy & 79.3 & & & \\
\hline Hemi or total glossectomy & 16.1 & $0.03^{*}$ & $6.10(1.34-27.64)$ & $0.02 *$ \\
\hline \multicolumn{5}{|l|}{ Lymph node dissection } \\
\hline SOND & 42.5 & & & \\
\hline MRND & 48.3 & $0.03^{*}$ & $1.65(0.53-5.14)$ & $0.04^{*}$ \\
\hline \multicolumn{5}{|l|}{ Reconstruction } \\
\hline No & 33.3 & & & \\
\hline Yes & 66.7 & 0.65 & & \\
\hline \multicolumn{5}{|l|}{ Tumor } \\
\hline $\mathrm{T} 1-2$ & 56.3 & & & \\
\hline T3-4 & 43.6 & 0.25 & & \\
\hline \multicolumn{5}{|l|}{ Node } \\
\hline No & 60.9 & & & \\
\hline N1-2 & 39.1 & $0.02 *$ & $2.44(0.73-8.15)$ & $0.03^{*}$ \\
\hline \multicolumn{5}{|l|}{ Radiotherapy } \\
\hline No & 46.0 & & & \\
\hline Yes & 54.0 & 0.77 & & \\
\hline \multicolumn{5}{|l|}{ Chemotherapy } \\
\hline No & 57.5 & & & \\
\hline Yes & 42.5 & 0.95 & & \\
\hline
\end{tabular}

HR, hazard ratio; CI, confidence interval; SOND, supraomohyoid neck dissection; MRND, modified radical neck dissection.

${ }^{*} \mathrm{p}<0.05$ by univariate and multivariate logistic regression analysis.

derwent MRND than in those who underwent SOND (HR, 1.652; 95\% CI, 0.53-5.14). The incidence of aspiration was 2.44 times higher in $\mathrm{N} 1$ and $\mathrm{N} 2$ patients than in patients with no metastasis (HR, 2.443; 95\% CI, 0.73-8.15).

When we conducted analyses of risk factors with aspiration in tongue cancer patients before surgery, there were no statistically significant risk factors. The results were the same when we conducted the analyses of risk factors in the full 133 patients.

\section{DISCUSSION}

This study intended to identify risk factors for dysphagia in patients with tongue cancer. To do this, we conducted a VFSS for the swallowing process and compared dysphagia characteristics before and after surgery. 
Tongue cancer patients experience difficulties in the pharyngeal phase of swallowing as well as in the oral phase. Patients who underwent VFSS after surgery had a higher incidence of inadequate tongue control and chewing, and delayed oral transit time in the oral phase. In the pharyngeal phase, aspiration and penetration, especially during fluid swallowing, were observed more frequently in the patients who underwent VFSS after surgery. Moreover, there were significant increases in inadequate laryngeal elevation, vallecular pouch residue, and pyriform sinus residue after surgery. In patients who VFSS was conducted both before and after surgery, significant differences in tongue control, chewing, oral transit time, laryngeal elevation, vallecular pouch residue, pyriform sinus residue, and aspiration and penetration were observed after surgery.

There were limitations because this data included patients who underwent VFSS only once. So, we compared VFSS findings from the patients performed both before and after surgery separately, too. The results showed that tongue control and oral transit time were more aggravated than other parameters in the oral phase. In the pharyngeal phase, aspiration and residue of the vallecular pouch were aggravated in particular.

Post-swallow residue is widely considered to be a sign of swallowing impairment and is assumed to pose a risk for aspiration on subsequent swallows. Post-swallow residue in one or both pharyngeal spaces was significantly associated with impaired swallowing safety on the subsequent clearing swallow for the same bolus [11].

Aspiration can be caused by numerous factors, including surgery, radiotherapy, chemotherapy, and abnormal motility of swallowing-related structures. Tumor resection can damage the structures that control swallowing, radiotherapy may cause scar tissue formation, and chemotherapy can result in abnormal motility of the muscles involved in swallowing due to cell apoptosis [12,13]. Aspiration was observed in $29.9 \%$ of tongue cancer patients in this study, which is lower than that seen in previous studies of HNC (36\%-94\%) [1,4,14-17]. This may be because HNC includes variable cancers related with anatomical structures involved during swallowing.

When food enters the oral cavity, the oral preparatory phase starts with manipulation of the bolus. Mechanoreceptor cells, which are concentrated on the tip of the tongue and the center of the palate, provide information concerning the position and size of the food bolus via the trigeminal nerve. The oral transport phase begins with peristaltic movements of the tongue that stimulate mechanoreceptors in the hard palate. The involuntary pharyngeal phase starts with the contraction of the genioglossus or mylohyoid muscles and involves the cooperative action of the suprahyoid muscles [18]. Aspiration was observed at a significantly higher rate in patients who underwent hemi- or total glossectomy than in those who underwent partial glossectomy. Previous studies have reported an incidence of aspiration in $10 \%-37 \%$ of patients who undergo a total glossectomy [19]; however, those studies did not evaluate differences according to the extent of surgery. Lango et al. [20] reported that tissue fibrosis and edema were aggravated after surgery. More extensive surgery leads to injury of structures related with swallowing, which leads to the occurrence of tissue fibrosis and edema.

Gender was significantly related to aspiration incidence in tongue cancer patients specifically. By contrast, in HNC overall, gender was not related to aspiration [3]. The correlation between gender and aspiration has not been broadly examined in the dysphagia field. Any biological explanation for this gender difference is absent. It is same, not only in the cancer population, but also in the general population [21]. In this study, male gender was an increased risk factor for aspiration in tongue cancer patients. Perhaps women are more assertive in requesting treatment and are more likely to receive a recommendation from their physician. Further research is needed to clarify the finding.

Chemotherapy is a principal predictive factor of aspiration in HNC. A systemic review demonstrated that reduced laryngeal excursion, base-of-tongue dysfunction, reduced pharyngeal contraction, and impaired epiglottic movement were most frequently reported [22]. However, in this study, there were no statistically significant differences in the incidence of aspiration between patients who received chemotherapy and those who did not. Radiotherapy was also not a predictive factor in tongue cancer patients. It will be necessary to compare radiotherapy and chemotherapy separately in a sufficient number of patients who received either chemotherapy or radiotherapy, because many patients received both in this study.

Patients with an N1 or N2 stage had a higher incidence of aspiration than patients with an N0 stage. Further- 
more, patients who underwent MRND had a higher incidence of aspiration than those who underwent SOND. Lymph node metastasis implies a more advanced disease, which requires more extensive resection. It can therefore be assumed that patients with a high nodal stage were more likely to have undergone MRND in this study.

The main limitation of this study was that only 28 patients underwent VFSS both before and after surgery. In addition, treatment modalities varied depending on tumor stage. Moreover, this study did not enroll all patients with tongue cancer, only those who were referred to our clinic for VFSS, which may introduce a selection bias. Further studies with a larger sample size should be performed in tongue cancer patients both before and after surgery.

In conclusion, tongue cancer patients experienced difficulties in the pharyngeal phase as well as the oral phase of swallowing. The difficulties were found to worsen after tongue cancer surgery. Male gender, extensive tumor resection, higher node stage, and more extensive lymph node dissection were major risk factors for aspiration in tongue cancer patients. Physicians should be aware of the characteristics of dysphagia in tongue cancer patients in order to effectively direct subsequent swallowing therapy.

\section{CONFLICT OF INTEREST}

No potential conflict of interest relevant to this article was reported.

\section{REFERENCES}

1. Nguyen NP, Frank C, Moltz CC, Vos P, Smith HJ, Bhamidipati PV, et al. Aspiration rate following chemoradiation for head and neck cancer: an underreported occurrence. Radiother Oncol 2006;80:302-6.

2. Nguyen NP, Moltz CC, Frank C, Vos P, Smith HJ, Nguyen $\mathrm{PD}$, et al. Impact of swallowing therapy on aspiration rate following treatment for locally advanced head and neck cancer. Oral Oncol 2007;43:352-7.

3. Jung SJ, Kim DY, Joo SY. Rick factors associated with aspiration in patients with head and neck cancer. Ann Rehabil Med 2011;35:781-90.

4. Kotz T, Costello R, Li Y, Posner MR. Swallowing dysfunction after chemoradiation for advanced squa- mous cell carcinoma of the head and neck. Head Neck 2004;26:365-72.

5. Namaki S, Tanaka T, Hara Y, Ohki H, Shinohara M, Yonhehara Y. Videofluorographic evaluation of dysphagia before and after modification of the flap and scar in patients with oral cancer. J Plast Surg Hand Surg 2011;45:136-42.

6. Logemann JA, Pauloski BR, Rademaker AW, McConnel FM, Heiser MA, Cardinale S, et al. Speech and swallow function after tonsil/base of tongue resection with primary closure. J Speech Hear Res 1993;36:91826.

7. Pauloski BR, Logemann JA, Rademaker AW, McConnel FM, Heiser MA, Cardinale S, et al. Speech and swallowing function after anterior tongue and floor of mouth resection with distal flap reconstruction. J Speech Hear Res 1993;36:267-76.

8. Han TR, Paik NJ, Park JW. The functional dysphagia scale using videofluoroscopic swallowing study in stroke patients. J Korean Acad Rehabil Med 1999;23:1118-26.

9. Pannunzio TG. Aspiration of oral feedings in patients with tracheostomies. AACN Clin Issues 1996;7:560-9.

10. Starmer H, Gourin C, Lua LL, Burkhead L. Pretreatment swallowing assessment in head and neck cancer patients. Laryngoscope 2011;121:1208-11.

11. Molfenter SM, Steele CM. The relationship between residue and aspiration on the subsequent swallow: an application of the normalized residue ratio scale. Dysphagia 2013;28:494-500.

12. Kikawada M, Iwamoto T, Takasaki M. Aspiration and infection in the elderly: epidemiology, diagnosis and management. Drugs Aging 2005;22:115-30.

13. Smith RV, Kotz T, Beitler JJ, Wadler S. Long-term swallowing problems after organ preservation therapy with concomitant radiation therapy and intravenous hydroxyurea: initial results. Arch Otolaryngol Head Neck Surg 2000;126:384-9.

14. Agarwal J, Palwe V, Dutta D, Gupta T, Laskar SG, Budrukkar A, et al. Objective assessment of swallowing function after definitive concurrent (chemo)radiotherapy in patients with head and neck cancer. Dysphagia 2011;26:399-406.

15. Carrara-de Angelis E, Feher O, Barros AP, Nishimoto IN, Kowalski LP. Voice and swallowing in patients enrolled in a larynx preservation trial. Arch Otolaryngol 
Head Neck Surg 2003;129:733-8.

16. Nguyen NP, Moltz CC, Frank C, Vos P, Smith HJ, Karlsson U, et al. Dysphagia following chemoradiation for locally advanced head and neck cancer. Ann Oncol 2004;15:383-8.

17. Steiniger JR, Parnes SM, Gardner GM. Morbidity of combined therapy for the treatment of supraglottic carcinoma: supraglottic laryngectomy and radiotherapy. Ann Otol Rhinol Laryngol 1997;106:151-8.

18. Plant RL. Anatomy and physiology of swallowing in adults and geriatrics. Otolaryngol Clin North Am 1998;31:477-88.

19. Weber RS, Ohlms L, Bowman J, Jacob R, Goepfert H. Functional results after total or near total glossectomy with laryngeal preservation. Arch Otolaryngol Head
Neck Surg 1991;117:512-5.

20. Lango MN, Egleston B, Ende K, Feigenberg S, D'Ambrosio DJ, Cohen RB, et al. Impact of neck dissection on long-term feeding tube dependence in patients with head and neck cancer treated with primary radiation or chemoradiation. Head Neck 2010;32:341-7.

21. Wilkins T, Gillies RA, Thomas AM, Wagner PJ. The prevalence of dysphagia in primary care patients: a HamesNet Research Network study. J Am Board Fam Med 2007;20:144-50.

22. Wall LR, Ward EC, Cartmill B, Hill AJ. Physiological changes to the swallowing mechanism following (chemo)radiotherapy for head and neck cancer: a systematic review. Dysphagia 2013;28:481-93. 